\title{
Critical care nurses' perception of their work and workload while taking care of the elderly patient in an Intensive care unit
}

\begin{abstract}
This qualitative descriptive exploratory case study aimed to investigate the perception of critical care nurses of their work and workload while caring for elderly patients in an intensive care unit. Eight Registered Nurses from an adult intensive care unit in a Brazilian University Hospital were interviewed, and the data collected were analyzed according to the content analysis. The findings show that nurses carry out some activities differently when patients are elderly. Still, they do not score it as additional activities in the Nursing Activities Score used for evaluating their nursing workload. They also recognized that the Nursing Activities Score does not reflect the real-time spend on the care of an elderly patient. This research may be noteworthy to nurses or organizations looking forward to enhancing their health care for elderly patients in critical care and improving the way nursing workload measurements acknowledging elderly patient needs.
\end{abstract}

Keywords: elderly care, critical care, nursing workload, intensive care units, nursing, complexity of care
Volume 6 Issue 2 - 2021

\author{
Meiriele Tavares Araujo,' Caroline Martins \\ Santos, ${ }^{2}$ Isabela Silva Cancio Velloso' \\ 'Applied Nursing Department, School of Nursing at the Federal \\ University of Minas Gerais, Brazil \\ ${ }^{2}$ Post-Graduation, School of Nursing at the Federal University of \\ Minas Gerais, Brazil
}

\begin{abstract}
Correspondence: Meiriele Tavares Araujo, Applied Nursing Department, School of Nursing at the Federal University of Minas Gerais, Brazil, Tel +55(43)9992-6945,
\end{abstract} Email meirieletavares@gmail.com

Received: February 18, 2021 | Published: March 03, 2021

\section{Introduction}

Intensive care units (ICU) are costly due to high technology and medical supplies, suitable infrastructure, and a highly trained team, the majority composed of nursing professionals. It can explain why the nursing workload discussion has been the focus of considerable interest and research for the last five decades. ${ }^{1-3}$ Besides that, the presence of elderly patients in the ICU increases their chances of needed interventions and severe instability. Furthermore, elderly patients may require intensive care for long periods than other adults..$^{4,5}$

Among the many tools to measure nursing workload in the literature and practice, the Nursing Activity Score (NAS) is considered the most frequently applied in the hospital setting, particularly in acute care units around the world, ${ }^{6-8}$ and it has been using by this study setting as well. NAS count nursing activities directly related to patients care and some other activities not directly related to them. The sum of its items scored reflects the amount of time spent by nursing staff to perform nursing care in a time frame of 24 hours. ${ }^{7}$

However, the relation between the workload measured by NAS, according to nursing care activities listed in the form and perceived by the nursing professional, is still under investigation in different settings and for different types of patients. ${ }^{8}$ Few studies discuss the relation of nursing workload and elderly patients, and the complex interface of ageing and other factors inherent to this population influences the workload needs more investigations. ${ }^{4,9}$ Considering this context, this study aimed to investigate the perception of critical care nurses of their work and workload while caring for elderly patients in an intensive care unit.

\section{Material and methods}

This study is a qualitative descriptive exploratory case study developed in the Adult Intensive Care Unit in a general university hospital in the southeast of Brazil. This unit has 14 mixed ICU beds (surgical, medical), although only 10 beds were active in the research period.
The unit nursing team had 32 nursing professionals, of which 1 are the nursing unit coordinator, 28 are bedside nurses and 3 licensed practitioner nursing (LPN). They are divided by team works with at least 5 nurses. There are two shift team, morning and afternoon team, which works for 6 hours each. Two nightshift teams work for 12 hours alternating evenings. Because of the high number of absences, frequently, there are not enough nurses for all beds, and they need to bring the LPN to work bedside under the nurse manager supervision.

The sample was designed by convenience and had at least one representative of each team shift. Eight nurses were interviewed, identified by the letter $\mathrm{N}$. These data were analyzed with the content analysis-oriented view based on nurses' perception of their work and workload when delivering care to elderly patients. The analysis depicted the two themes difference between caring for elderly patients in an acute setting and the perception of the NAS scored for elderly and adult patients.

The Ethics Committee of the Federal University of Minas Gerais approved this study under report number 3059.907/2018.

\section{Findings and discussion}

The participants were 8 nurses, five of them female, aged from 28 to 49 -years-old, working in the unit between 1,6 and 5 years. They finished their nursing program between 4 and 13 years ago, and only 1 of them did not have a post-graduation course. Most of them took emergency and critical care course. These nurses had a 36-hours work journey per week in the ICU.

\section{The difference between caring for elderly patients in an acute setting}

When questioned about differences in delivering care to an adult or elderly patient in the ICU, most nurses said they need to act differently, sometimes showing more affection, staying closer to them longer, listening to their demands and stories. Also, they need to perform with more precautions some basics nursing activities such as hygiene care, 
application of risk assessment tools, oral feeding, mobilizations and positioning, and others.

“...when this is an elderly person, I try to treat him with more affection more warmth. I try to listen to a few his pains, lamentations, fears..."(N1)

“...elderly patient demands little more than adults, right?... sometimes you must give them more support, pay more attention in listening to them, to listen differently, right?... They want to tell a bigger story than younger adults...”(N2)

"...They have more fragile skin, so their hygiene procedures must be differentiated to avoid cause injures...The elderly patient is usually confused and has a higher risk of falls, so it demands more monitoring. The elderly's feeding must be assisted due to the risk of aspiration in the elderly patient is much higher". (N6)

"...In fact, they demand more. They demand more strength, more attention, more protection during procedures such as mobilization, hygiene, and feeding, which rely on their condition to be totally dependent, right? And so, it is their particularities...due to the natural process of ageing. They demand more due to organic and physical limitations". (N8)

The precautions during the care presented by the nurses are based on their concern about the ageing process that decreases the physiologic organ reserve and tolerance to treatment and raises the chances of adverse events may occur and compromised patient safety. Furthermore, the heterogeneous ageing process reinforces the necessity of practising individualized care, which means that critical care nurses need to be more aware of the gerontological features of ageing in their clinical routine. ${ }^{9,10}$

\section{The perception of the NAS scored for elderly and adult patients}

Nurses usually don't compare the NAS scored with the patient care delivered. Some nurses said that being elderly or adult does not interfere with the NAS results because it is more related to patient complexity. We inferred that they don't perceive an elderly patient as a complex patient. However, some noted that elderly patients required more bedside presence and more time to developing some of the nurses' basic care.

"...we don't have the habit of always looking at which patient has the bigger NAS... Well, I never stopped to observe this also. But probably if you narrow it down well, you will see that the elderly might have more demands, right, but... I never observed (N2)"

"...No, honestly? No. Sometimes, it is so busy that this close glance - thinking about the patient who is more dependent and making a comparison with the NAS itself. Unfortunately, I don't think we do so. It depends a lot on the professional, the shift day, the routine; honestly, I don't think so. On a daily basis, I realize that sometimes there is a difference in the final sum, but it is not a constant (N5)"

"...The elderly require a more attentive look, but in terms of NAS and workload, it is not for all elderly...I think it depends a lot on the clinical condition of the patient. I believe the patient's clinical condition has more influence on NAS score than the fact of being elderly or not (N9)“

According to the nurses' perception, the NAS scored reflects more the patients' complexity than how the elderly patient needs to be assisted. Also, another study reports that in ICU, the care of older people, compared to the adult ones, is correlated with a rise in nursing workload, which means that ageing can be considered an associated factor for nursing workload, but not a predictor of it. ${ }^{4,9}$ Following this idea, taking care of an elderly patient requires slowing down and spending more time in the care activities than the number of activities done which is not considered in the NAS.

"...I think it depends a lot on an elderly patient because there are needs not assessed by the NAS. Such as demands of emotional support, talking, requesting all the time, sometimes asking just for having someone closer, and NAS cannot assess these. However, these demands required a lot from the professional who is with the patient. If I am taking care of two patients, I cannot be with one all the time. I cannot sit next to him and talk, hold his hand, and chat. I can't because I have another patient to take care of, and sometimes in more critical condition... Older patient like to please others and don't like or are afraid of bothering the nurses, and because of that, they don't request something. For example, a patient who has diuresis on a bedpan doesn't ask to put the bedpan on not to bother the nurses, and then it ends up giving him bladder distension. So, I think these are things that those who delivery the assistance knows, who see the demand, whether they want it or not. There is no way to measure this in NAS...And the fact that we are dividing our attention when we are not with only one patient it impossible for us to dedicate more attention to these elderly needs. (N9)"

It was also mentioned that they want to have more time to give the elderly patients more dedicated care, but their heavy workload often doesn't allow them to do it the way they would like to do. This perception aligned their desire to another study that advocated a care model in acute care for elders. The recognition of patient-centred care may contribute to restoring their physical functioning and mental health condition. ICU hospitalization is well-known as a hostile (unsafe and anxiety-producing) physical environment and processes of patient care (e.g., restricting mobility, isolation) $)^{5}$. Additionally, it is necessary to assure that when an older patient is admitted to the ICU, they must receive the most appropriate treatment, which sometimes doesn't mean the highest treatment unbalanced to their chances of survival, but a shared decision-making process about certain treatments available and close communication with family and other caregivers. ${ }^{5,10}$

\section{Conclusion}

The nurses have a perception that elderly patient demands more in ICU everyday work, and it is impossible for the NAS to capture some of the elderly real needs. The workload increases not only because of the number or complexity of activities but also because of the duration and how it is performed. This nurses also understand it as crucial to show more affection and attention for the elderly patient. The NAS might demand being adapted to the time required for assisting an elderly patient in critical conditions, not only for their complexity.

\section{Acknowledgments}

We want to express our sincere gratitude to all nurses who contributed to this research and thank the university hospital's Nursing Director office for all support to accomplish this study.

\section{Conflicts of interest}

The authors declare that there is no conflict of interest.

\section{References}

1. Mildon BL. The concept of home care nursing workload: analysis and significance. Published online 2011. 
2. Alghamdi MG. Nursing workload: a concept analysis. J Nurs Manag 2016;24(4):449-457.

3. Griffiths P, Saville C, Ball J, et al. Nursing workload, nurse staffing methodologies and tools: a systematic scoping review and discussion. Int J Nurs Stud. 2020;103:103487.

4. Sousa RMC de, Padilha KG, Nogueira L de S, et al. Carga de trabalho de enfermagem requerida por adultos, idosos e muito idosos em Unidade de Terapia Intensiva. Rev da Esc Enferm da USP. 2009;43(spe2):1284-1291.

5. Palmer RM. geriatrics the acute care for elders unit model of care. 2018.

6. Bruyneel A, Tack J, Droguet M, et al. Measuring the nursing workload in intensive care with the Nursing Activities Score (NAS): A prospective study in 16 hospitals in Belgium. J Crit Care. 2019;54:205-211.
7. Padilha KG, Stafseth S, Solms D, et al. Nursing activities score: An updated guideline for its application in the intensive care unit. Rev da Esc Enferm. 2015;49(SpecialIssue):131-137.

8. Ferreira SC, Santos MJ de OL, Estrela FM. Nursing activities score e o cuidado em uma unidade de terapia intensiva. Arq Ciências da Saúde. 2016;23(1):63.

9. Ferretti-Rebustini RE de L, Nogueira L de S, Silva R de CG e, et al. Aging as a predictor of nursing workload in intensive care Unit: results from a Brazilian Sample. Rev Esc Enferm USP. 2017;51:e03216-e03216.

10. Guidet B, Vallet H, Boddaert J, et al. Caring for the critically ill patients over 80: a narrative review. Ann Intensive Care. 2018;8:114. 